\title{
Generalized Derivations on Prime Near Rings
}

\author{
Asma Ali, ${ }^{1}$ Howard E. Bell, ${ }^{2}$ and Phool Miyan' \\ ${ }^{1}$ Department of Mathematics, Aligarh Muslim University, Aligarh 202002, India \\ ${ }^{2}$ Department of Mathematics, Brock University, St. Catharines, ON, Canada L2S 3A1
}

Correspondence should be addressed to Asma Ali; asma.ali345@gmail.com

Received 8 October 2012; Accepted 2 January 2013

Academic Editor: Christian Corda

Copyright (C) 2013 Asma Ali et al. This is an open access article distributed under the Creative Commons Attribution License, which permits unrestricted use, distribution, and reproduction in any medium, provided the original work is properly cited.

\begin{abstract}
Let $N$ be a near ring. An additive mapping $f: N \rightarrow N$ is said to be a right generalized (resp., left generalized) derivation with associated derivation $d$ on $N$ if $f(x y)=f(x) y+x d(y)$ (resp., $f(x y)=d(x) y+x f(y)$ ) for all $x, y \in N$. A mapping $f: N \rightarrow N$ is said to be a generalized derivation with associated derivation $d$ on $N$ if $f$ is both a right generalized and a left generalized derivation with associated derivation $d$ on $N$. The purpose of the present paper is to prove some theorems in the setting of a semigroup ideal of a 3-prime near ring admitting a generalized derivation, thereby extending some known results on derivations.
\end{abstract}

\section{Introduction}

Throughout the paper, $N$ denotes a zero-symmetric left near ring with multiplicative center $Z$, and for any pair of elements $x, y \in N,[x, y]$ denotes the commutator $x y-y x$, while the $\operatorname{symbol}(x, y)$ denotes the additive commutator $x+y-x-y$. A near ring $N$ is called zero-symmetric if $0 x=0$, for all $x \in N$ (recall that left distributivity yields that $x 0=0$ ). The near ring $N$ is said to be 3-prime if $x N y=\{0\}$ for $x, y \in N$ implies that $x=0$ or $y=0$. A near ring $N$ is called 2-torsion-free if $(N,+)$ has no element of order 2. A nonempty subset $A$ of $N$ is called a semigroup right (resp., semigroup left) ideal if $A N \subseteq A$ (resp., $N A \subseteq A$ ), and if $A$ is both a semigroup right ideal and a semigroup left ideal, it is called a semigroup ideal. An additive mapping $d: N \rightarrow N$ is a derivation on $N$ if $d(x y)=d(x) y+$ $x d(y)$, for all $x, y \in N$. An additive mapping $f: N \rightarrow N$ is said to be a right (resp., left) generalized derivation with associated derivation $d$ if $f(x y)=f(x) y+x d(y)$ (resp., $f(x y)=d(x) y+x f(y))$, for all $x, y \in N$, and $f$ is said to be a generalized derivation with associated derivation $d$ on $N$ if it is both a right generalized derivation and a left generalized derivation on $N$ with associated derivation $d$. (Note that this definition differs from the one given by Hvala in [1]; his generalized derivations are our right generalized derivations.) Every derivation on $N$ is a generalized derivation.

In the case of rings, generalized derivations have received significant attention in recent years. We prove some theorems in the setting of a semigroup ideal of a 3-prime near ring admitting a generalized derivation and thereby extend some known results [2, Theorem 2.1], [3, Theorem 3.1], [4, Theorem 3], and [5, Theorem 3.3].

\section{Preliminary Results}

We begin with several lemmas, most of which have been proved elsewhere.

Lemma 1 (see [3, Lemma 1.3]). Let $N$ be a 3-prime near ring and $d$ be a nonzero derivation on $N$.

(i) If $U$ is a nonzero semigroup right ideal or a nonzero semigroup left ideal of $N$, then $d(U) \neq\{0\}$.

(ii) If $U$ is a nonzero semigroup right ideal of $N$ and $x$ is an element of $N$ which centralizes $U$, then $x \in Z$.

Lemma 2 (see [3, Lemma 1.2]). Let $\mathrm{N}$ be a 3-prime near ring.

(i) If $z \in Z \backslash\{0\}$, then $z$ is not a zero divisor.

(ii) If $Z \backslash\{0\}$ contains an element $z$ for which $z+z \in Z$, then $(N,+)$ is abelian.

(iii) If $z \in Z \backslash\{0\}$ and $x$ is an element of $N$ such that $x z \in Z$, then $x \in Z$. 
Lemma 3 (see [3, Lemmas 1.3 and 1.4]). Let $N$ be a 3-prime near ring and $U$ be a nonzero semigroup ideal of $N$. Let $d$ be a nonzero derivation on $N$.

(i) If $x, y \in N$ and $x U y=\{0\}$, then $x=0$ or $y=0$.

(ii) If $x \in N$ and $x U=\{0\}$ or $U x=\{0\}$, then $x=0$.

(iii) If $x \in N$ and $d(U) x=\{0\}$ or $x d(U)=\{0\}$, then $x=0$.

Lemma 4 (see [3, Lemma 1.5]). If $N$ is a 3-prime near ring and $Z$ contains a nonzero semigroup left ideal or a semigroup right ideal, then $N$ is a commutative ring.

Lemma 5. If $f$ is a generalized derivation on $N$ with associated derivation $d$, then $(d(x) y+x f(y)) z=d(x) y z+x f(y) z$, for all $x, y, z \in N$.

Proof. We prove only (ii), since (i) is proved in [2]. For all $x, y, z \in N$ we have $f((x y) z)=f(x y) z+x y d(z)=$ $(d(x) y+x f(y)) z+x y d(z)$ and $f(x(y z))=d(x) y z+x f(y z)=$ $d(x) y z+x f(f(y) z+y d(z))=d(x) y z+x f(y) z+x y d(z)$. Comparing the two expressions for $f(x y z)$ gives (ii).

Lemma 6. Let $N$ be a 3-prime near ring and $f$ a generalized derivation with associated derivation $d$.

(i) $f(x) y+x d(y)=x d(y)+f(x) y$ for all $x, y \in N$.

(ii) $d(x) y+x f(y)=x f(y)+d(x) y$ for all $x, y \in N$.

Proof. (i) $f(x(y+y))=f(x)(y+y)+x d(y+y)=f(x) y+$ $f(x) y+x d(y)+x d(y)$, and $f(x y+x y)=f(x) y+x d(y)+$ $f(x) y+x d(y)$. Comparing these two equations gives the desired result.

(ii) Again, calculate $f(x(y+y))$ and $f(x y+x y)$ and compare.

Lemma 7. Let $N$ be a 3-prime near ring and $f$ a generalized derivation with associated derivation $d$. Then $f(Z) \subseteq Z$.

Proof. Let $z \in Z$ and $x \in N$. Then $f(z x)=f(x z)$; that is, $f(z) x+z d(x)=d(x) z+x f(z)$. Applying Lemma 6(i), we get $z d(x)+f(z) x=d(x) z+x f(z)$. It follows that $f(z) x=x f(z)$ for all $x \in N$, so $f(z) \in Z$.

Lemma 8. Let $N$ be a 3-prime near ring and $U$ a nonzero semigroup ideal of $N$. If $f$ is a nonzero right generalized derivation of $N$ with associated derivationd, then $f(U) \neq\{0\}$.

Proof. Suppose $f(U)=\{0\}$. Then $f(u x)=f(u) x+u d(x)=$ $0=u d(x)$ for all $u \in U$ and $x \in N$, and it follows by Lemma 3(ii) that $d=0$. Therefore $f(x u)=f(x) u=0$ for all $u \in U$ and $x \in N$, and another appeal to Lemma 3(ii) gives $f=0$, which is a contradiction.

Lemma 9 (see [2, Theorem 2.1]). Let $\mathrm{N}$ be a 3-prime near ring with a nonzero right generalized derivation $f$ with associated derivation d. If $f(N) \subseteq Z$ then $(N,+)$ is abelian. Moreover, if $N$ is 2-torsion-free, then $N$ is a commutative ring.

Lemma 10 (see [2, Theorem 4.1]). Let $N$ be a 2-torsion-free 3-prime near ring and $f$ a nonzero generalized derivation on
$N$ with associated derivation $d$. If $[f(N), f(N)]=\{0\}$, then $N$ is a commutative ring.

\section{Main Results}

The theorems that we prove in this section extend the results proved in [2, Theorems 2.1 and 3.1], [3, Theorems 2.1, 3.1, and 3.3], and [5, Theorem 3.3].

Theorem 11. Let $N$ be a 3-prime near ring and $U$ be a nonzero semigroup ideal of $N$. Let $f$ be a nonzero right generalized derivation with associated derivation $d$. If $f(U) \subseteq Z$, then $(N,+)$ is abelian. Moreover, if $N$ is 2-torsion-free, then $N$ is a commutative ring.

Proof. We begin by showing that $(N,+)$ is abelian, which by Lemma 2(ii) is accomplished by producing $z \in Z \backslash\{0\}$ such that $z+z \in Z$. Let $a$ be an element of $U$ such that $f(a) \neq 0$. Then for all $x \in N, a x \in U$ and $a x+a x=a(x+x) \in U$, so that $f(a x) \in Z$ and $f(a x)+f(a x) \in Z$; hence we need only to show that there exists $x \in N$ such that $f(a x) \neq 0$. Suppose that this is not the case, so that $f((a x) a)=0=f(a x) a+a x d(a)=$ $\operatorname{axd}(a)$, for all $x \in N$. By Lemma 3(i) either $a=0$ or $d(a)=0$.

If $d(a)=0$, then $f(x a)=f(x) a+x d(a)$; that is, $f(x a)=$ $f(x) a \in Z$, for all $x \in N$. Thus $[f(u) a, y]=0$, for all $y \in N$, and $u \in U$. This implies that $f(u)[a, y]=0$, for all $u \in U$ and $y \in N$ and Lemma 2(i) gives $a \in Z$. Thus $0=f(a x)=$ $f(x a)=f(x) a$, for all $x \in N$. Replacing $x$ by $u \in U$, we have $f(U) a=0$, and by Lemmas 2(i) and 8, we get that $a=0$. Thus, we have a contradiction.

To complete the proof, we show that if $N$ is 2-torsion-free, then $N$ is commutative.

Consider first the case $d=0$. This implies that $f(u x)=$ $f(u) x \in Z$ for all $u \in U$ and $x \in N$. By Lemma 8, we have $u \in U$ such that $f(u) \in Z \backslash\{0\}$, so $N$ is commutative by Lemma 2(iii).

Now consider the case $d \neq 0$. Let $c \in Z \backslash\{0\}$. This implies that if $x \in U, f(x c)=f(x) c+x d(c) \in Z$. Thus $(f(x) c+$ $x d(c)) y=y(f(x) c+x d(c))$ for all $x, y \in U$ and $c \in Z$. Therefore by Lemma 5(i), $f(x) c y+x d(c) y=y f(x) c+y x d(c)$ for all $x, y \in U$ and $c \in Z$. Since $d(c) \in Z$ and $f(x) \in Z$, we obtain $d(c)[x, y]=0$, for all $x, y \in U$ and $c \in Z$. Let $d(Z) \neq 0$. Choosing $c$ such that $d(c) \neq 0$ and noting that $d(c)$ is not a zero divisor, we have $[x, y]=0$ for all $x, y \in U$. By Lemma 1(ii), $U \subseteq Z$; hence $N$ is commutative by Lemma 4 .

The remaining case is $d \neq 0$ and $d(Z)=\{0\}$. Suppose we can show that $U \cap Z \neq\{0\}$. Taking $z \in(U \cap Z) \backslash\{0\}$ and $x \in$ $N$, we have $f(x z)=f(x) z \in Z$; therefore $f(N) \subseteq Z$ by Lemma 2(iii) and $N$ is commutative by Lemma 9 .

Assume, then, that $U \cap Z=\{0\}$. For each $u \in U, f\left(u^{2}\right)=$ $f(u) u+u d(u)=u(f(u)+d(u)) \in U \cap Z$, so $f\left(u^{2}\right)=0$. Thus, for all $u \in U$ and $x \in N, f\left(u^{2} x\right)=f\left(u^{2}\right) x+u^{2} d(x)=$ $u^{2} d(x) \in U \cap Z$, so $u^{2} d(x)=0$, and by Lemma 3(iii) $u^{2}=0$. Since $f(x u)=f(x) u+x d(u) \in Z$ for all $u \in U$ and $x \in N$, we have $(f(x) u+x d(u)) u=u(f(x) u+x d(u))$, and right multiplying by $u$ gives $u x d(u) u=0$. Consequently $d(u) u N d(u) u=\{0\}$, so that $d(u) u=0$ for all $u \in U$. Since $u^{2}=0, d\left(u^{2}\right)=d(u) u+u d(u)=0$ for all $u \in U$, so 
$f(u) u=0$ for all $u \in U$. But by Lemma 8, there exists $u_{0} \in U$ for which $f\left(u_{0}\right) \neq 0$; and since $f\left(u_{0}\right) \in Z$, we get $u_{0}=0-\mathrm{a}$ contradiction. Therefore $U \cap Z \neq\{0\}$ as required.

Theorem 12. Let $N$ be a 3-prime near ring with a nonzero generalized derivation $f$ with associated nonzero derivation $d$. Let $U$ be a nonzero semigroup ideal of $N$. If $[f(U), f(U)]=0$, then $(N,+)$ is abelian.

Proof. Assume that $x \in N$ is such that $[x, f(U)]=[x+$ $x, f(U)]=0$. For all $u, v \in U$ such that $u+v \in U$, $[x+x, f(u+v)]=0$.

This implies that

$$
\begin{gathered}
(x+x) f(u+v)=f(u+v)(x+x), \\
(x+x) f(u)+(x+x) f(v) \\
=f(u+v) x+f(u+v) x, \\
f(u)(x+x)+f(v)(x+x) \\
=x f(u+v)+x f(u+v), \\
f(u) x+f(u) x+f(v) x+f(v) x \\
=x f(u)+x f(v)+x f(u)+x f(v), \\
x f(u)+x f(u)+x f(v)+x f(v) \\
=x f(u)+x f(v)+x f(u)+x f(v), \\
x(f(u)+f(v)-f(u)-f(v))=0, \\
x f(u+v-u-v)=0 .
\end{gathered}
$$

This equation may be restated as $x f(c)=0$, where $c=(u, v)$.

Let $a, b \in U$. Then $a b \in U$ and $a b+a b=a(b+b) \in U$, so $[f(a b), f(U)]=\{0\}=[f(a b)+f(a b), f(U)]$, and by the argument in the previous paragraph, $f(a b) f(c)=0$. We now have $f\left(U^{2}\right) f(u, v)=0$ for all $u, v \in U$ such that $u+v \in U$. Taking $w \in U^{2}$ and $x \in N$, we get $f(x w) f((u, v))=$ $(d(x) w+x f(w)) f((u, v))=0=d(x) w f((u, v))$, and since $U^{2}$ is a nonzero semigroup ideal by Lemma 3(ii) and $d \neq 0$, Lemma 3(i) gives

$$
f((u, v))=0 \quad \forall u, v \in U \text { such that } u+v \in U .
$$

Take $u=r y$ and $v=r z$, where $r \in U$ and $y, z \in N$, so that $u+v=r y+r z=r(y+z) \in U$. By (2) we have

$$
f((r y, r z))=f(r(y, z))=0 \quad \forall r \in U, y, z \in N .
$$

Replacing $r$ by $r w, w \in U$, we obtain $f(r(w y, w z))=0=$ $d(r)(w(y, z))+r f((w y, w z))$; so by $(3) d(r) U(y, z)=0$ for all $r \in U$ and $y, z \in N$. It follows immediately by Lemmas 1(i) and 3(i) that $(y, z)=0$ for all $y, z \in N$; that is, $(N,+)$ is abelian.

Theorem 13. Let $N$ be a 2-torsion-free 3-prime near ring and $U$ be a nonzero semigroup ideal of $N$. If $f$ is a nonzero generalized derivation with associated derivation $d$ such that $[f(U), f(U)]=0$, then $N$ is a commutative ring if it satisfies one of the following: (i) $d(Z) \neq\{0\}$; (ii) $U \cap Z \neq\{0\}$; (iii) $d=0$ and $f(Z) \neq\{0\}$.

Proof. (i) Let $a \in N$ centralizes $f(U)$, and let $z \in Z$ such that $d(z) \neq 0$. Then a centralizes $f(u z)$ for all $u \in U$, so that $a(f(u) z+u d(z))=(f(u) z+u d(z)) a$ and $\operatorname{aud}(z)=u d(z) a$. Since $d(z) \in Z \backslash\{0\}, d(z)[a, u]=0=[a, u]$ for all $u \in U$. Therefore a centralizes $U$, and by Lemma 1(ii), $a \in Z$. Since $f(U)$ centralizes $f(U), f(U) \subseteq Z$ and our result follows by Theorem 11.

(ii) We may assume $d(Z)=\{0\}$. Let $z \in(U \cap Z) \backslash\{0\}$. Then for all $x, y \in N, f(x z)=f(x) z$ and $f(y z)=f(y) z$ commute; hence $z^{2}[f(x), f(y)]=0=[f(x), f(y)]$. Our result now follows from Lemma 10.

(iii) Let $u, v \in U$ and $z \in Z$ such that $f(z) \neq 0$. Then $[f(z u), f(u)]=0=[f(z) u, f(v)]$, and since $f(z) \in Z \backslash\{0\}$, $f(z)[u, f(u)]=0=[u, f(v)]$. Thus $f(U)$ centralizes $U$, and by Lemma 1(ii), $f(U) \subseteq Z$. Our result now follows by Theorem 11.

We have already observed that if $f$ is a generalized derivation with $d=0$, then $f(x) y=x f(y)$ for all $x, y \in N$. For 3-prime near rings, we have the following converse.

Theorem 14. Let $N$ be a 3-prime near ring and $U$ be a nonzero semigroup ideal of $N$. If $f$ is a nonzero right generalized derivation of $N$ with associated derivation $d$ and $f(x) y=$ $x f(y)$, for all $x, y \in U$, then $d=0$.

Proof. We are given that $f(x) y=x f(y)$ for all $x, y \in U$. Substituting $y z$ for $y$, we get $f(x) y z=x f(y z)=x(f(y) z+$ $y d(z))$ for all $x, y, z \in U$. It follows that $x y d(z)=0$ for all $x, y, z \in U$; that is, $x U d(z)=\{0\}$ for all $x, z \in U$. By Lemma 3(i), $d(U)=0$, and hence $d=0$ by Lemma 1(i).

\section{Generalized Derivations Acting as a Homomorphism or an Antihomomorphism}

In [4], Bell and Kappe proved that if $R$ is a semiprime ring and $d$ is a derivation on $R$ which is either an endomorphism or an antiendomorphism on $R$, then $d=0$. Of course, derivations which are not endomorphisms or antiendomorphisms on $R$ may behave as such on certain subsets of $R$; for example, any derivation $d$ behaves as the zero endomorphism on the subring $C$ consisting of all constants (i.e., the elements $x$ for which $d(x)=0$ ). In fact in a semiprime $\operatorname{ring} R, d$ may behave as an endomorphism on a proper ideal of $R$. However as noted in [4], the behaviour of $d$ is somewhat restricted in the case of a prime ring. Recently the authors in [6] considered $(\theta, \phi)$-derivation $d$ acting as a homomorphism or an antihomomorphism on a nonzero Lie ideal of a prime ring and concluded that $d=0$. In this section we establish similar results in the setting of a semigroup ideal of a 3-prime near ring admitting a generalized derivation.

Theorem 15. Let $N$ be a 3-prime near ring and $U$ be a nonzero semigroup ideal of $N$. Let $f$ be a nonzero generalized derivation on $N$ with associated derivation $d$. If $f$ acts as a 
homomorphism on $U$, then $f$ is the identity map on $N$ and $d=0$.

Proof. By the hypothesis

$$
f(x y)=d(x) y+x f(y)=f(x) f(y) \quad \forall x, y \in U .
$$

Replacing $y$ by $y z$ in the above relation, we get

$$
f(x y z)=d(x) y z+x f(y z) \quad \forall x, y, z \in U,
$$

or

$$
\begin{array}{r}
f(x y) f(z)=d(x) y z+x(d(y) z+y f(z)) \\
\forall x, y, z \in U .
\end{array}
$$

This implies that

$$
\begin{array}{r}
(d(x) y+x f(y)) f(z)=d(x) y z+x d(y) z+x y f(z) \\
\forall x, y, z \in U .
\end{array}
$$

Using Lemma 5(ii), we get

$$
\begin{aligned}
& d(x) y f(z)+x f(y) f(z) \\
& \quad=d(x) y z+x d(y) z+x y f(z) \quad \forall x, y, z \in U
\end{aligned}
$$

or

$$
\begin{aligned}
& d(x) y f(z)+x f(y z) \\
& \quad=d(x) y z+x d(y) z+x y f(z) \quad \forall x, y, z \in U .
\end{aligned}
$$

This implies that

$$
\begin{aligned}
& d(x) y f(z)+x d(y) z+x y f(z) \\
& \quad=d(x) y z+x d(y) z+x y f(z) \quad \forall x, y, z \in U .
\end{aligned}
$$

That is,

$$
d(x) y f(z)=d(x) y z \quad \forall x, y, z \in U
$$

Therefore

$$
d(x) y(f(z)-z)=0 \quad \forall x, y, z \in U,
$$

which implies that

$$
d(x) U(f(z)-z)=\{0\} \quad \forall x, z \in U .
$$

It follows by Lemma 3(i) that either $d(U)=0$ or $f(z)=z$ for all $z \in U$.

In fact, as we now show, both of these conditions hold.

Suppose that $f(u)=u$ for all $u \in U$. Then for all $u \in U$ and $x \in N, f(x u)=x u=d(x) u+x f(u)=d(x) u+x u$; hence $d(x) U=\{0\}$ for all $x \in N$, and thus $d=0$.

On the other hand, suppose that $d(U)=\{0\}$, so that $d=0$. Then for all $x, y \in U, f(x y)=f(x) y=f(x) f(y)$, so that $f(x)(y-f(y))=0$. Replacing $y$ by $z y, z \in N$, and noting that $f(z y)=z f(y)$, we see that $f(x) N(y-f(y))=\{0\}$ for all $x, y \in U$. Therefore, $f(U)=\{0\}$ or $f$ is the identity on $U$. But $f(U)=\{0\}$ contradicts Lemma 8 , so $f$ is the identity on $U$.

We now know that $f$ is the identity on $U$ and $f(x y)=$ $x f(y)$ for all $x, y \in N$. Consequently, $f(u x)=u x=u f(x)$ for all $u \in U$ and $x \in N$, so that $U(x-f(x))=\{0\}$ for all $x \in N$. It follows that $f$ is the identity on $N$.

Theorem 16. Let $N$ be a 3-prime near ring and $U$ be a nonzero semigroup ideal of $N$. If $f$ is a nonzero generalized derivation on $N$ with associated derivation $d$. If $f$ acts as an antihomomorphism on $U$, then $d=0, f$ is the identity map on $N$, and $N$ is a commutative ring.

Proof. We begin by showing that $d=0$ if and only if $f$ is the identity map on $N$.

Clearly if $f$ is the identity map on $N, x d(y)=0$ for all $x, y \in N$, and hence $d=0$.

Conversely, assume that $d=0$, in which case $f(x y)=$ $f(x) y=x f(y)$ for all $x, y \in N$. It follows that for any $x, y, z \in$ $U$,

$$
\begin{aligned}
f(y x z) & =f(z) f(y x)=f(z) y f(x) \\
& =f(z y) f(x)=z f(y) f(x)=z f(x y) .
\end{aligned}
$$

On the other hand,

$$
\begin{aligned}
f(y x z) & =f(x z) f(y)=f(x) z f(y)=f(x) f(z y) \\
& =f(x) f(y) f(z)=f(y x) f(z)=f(y) x f(z) \\
& =f(y) f(x z)=f(y) f(x) z=f(x y) z .
\end{aligned}
$$

Comparing (14) and (15) shows that $f\left(U^{2}\right)$ centralizes $U$, so that $f\left(U^{2}\right) \subseteq Z$ by Lemma 1(ii).

Now $U^{2}$ is a nonzero semigroup ideal by Lemma 3(ii); hence $f\left(U^{2}\right) \neq 0$ by Lemma 8 . Choosing $x, y \in U$ such that $f(x y) \neq 0$, we see that for any $z \in U, f(x y) z=f(x y z)=$ $f(y z) f(x)=f(y) z f(x)=f(y) f(z x)=f(y) f(x) f(z)=$ $f(x y) f(z)$, and hence $f(x y)(z-f(z))=0$. Since $f(x y) \in$ $Z \backslash\{0\}$, we conclude that $f(z)=z$ for all $z \in U$, and it follows easily that $f$ is the identity map on $N$.

We note now that if the identity map on $N$ acts as an antihomomorphism on $U$, then $U$ is commutative, so that by Lemmas 1 (ii) and $4 \mathrm{~N}$ is a commutative ring.

To complete the proof of our theorem, we need only to argue that $d=0$. By our antihomomorphism hypothesis

$$
f(x y)=d(x) y+x f(y)=f(y) f(x) \quad \forall x, y \in U .
$$

Replacing $y$ by $x y$ in the above relation, we get

$$
f(x y) f(x)=f(x x y)=d(x) x y+x f(x y) \quad \forall x, y \in U .
$$

This implies that

$$
\begin{aligned}
& (d(x) y+x f(y)) f(x) \\
& \quad=d(x) x y+x f(y) f(x) \quad \forall x, y \in U
\end{aligned}
$$


Using Lemma 5(ii), we get

$$
\begin{aligned}
& d(x) y f(x)+x f(y) f(x) \\
& \quad=d(x) x y+x f(y) f(x) \quad \forall x, y \in U .
\end{aligned}
$$

Thus

$$
d(x) y f(x)=d(x) x y \quad \forall x, y \in U .
$$

Replacing $y$ by $y r$ in (20) and using (20), we get

$$
\begin{array}{r}
d(x) \operatorname{yrf}(x)=d(x) x y r, \text { and so } d(x) y[r, f(x)]=0 \\
\forall x, y \in U, r \in N .
\end{array}
$$

Application of Lemma 3(i) yields that for each $x \in U$ either $d(x)=0$ or $[r, f(x)]=0$; that is $d(x)=0$ or $f(x) \in Z$.

Suppose that there exists $w \in U$ such that $f(w) \in Z \backslash\{0\}$. Then for all $v \in U$ such that $d(v)=0, f(w v)=f(w) v=$ $f(v) f(w)=f(w) f(v)$, and hence $f(w)(v-f(v))=0=$ $v-f(v)$. Now consider arbitrary $x, y \in U$. If one of $f(x), f(y)$ is in $Z$, then $f(x y)=f(x) f(y)$. If $d(x)=0=d(y)$, then $d(x y)=d(x) y+x d(y)=0$, so $f(x y)=x y=f(x) f(y)$. Therefore $f(x y)=f(x) f(y)$ for all $x, y \in U$, and by Theorem 15, $f$ is the identity map on $N$, and therefore $d=0$.

The remaining possibility is that for each $x \in U$, either $d(x)=0$ or $f(x)=0$. Let $u \in U \backslash\{0\}$, and let $U_{1}=u N$. Then $U_{1}$ is a nonzero semigroup right ideal contained in $U$ and $U_{1}$ is an additive subgroup of $N$. The sets $\left\{x \in U_{1} \mid d(x)=0\right\}$ and $\left\{x \in U_{1} \mid f(x)=0\right\}$ are additive subgroups of $U_{1}$ with union equal to $U_{1}$, so $d\left(U_{1}\right)=\{0\}$ or $f\left(U_{1}\right)=\{0\}$. If $d\left(U_{1}\right)=\{0\}$, then $d=0$ by Lemma 1(i). Suppose, then, that $f\left(U_{1}\right)=\{0\}$. Then for arbitrary $x, y \in N f(u x y)=f(u x) y+u x d(y)=0=$ $u x d(y)$, so $u N d(y)=\{0\}$, and again $d=0$. This completes the proof.

\section{Acknowledgments}

A. Ali and P. Miyan gratefully acknowledge the support received by the University Grants Commission, India Grant F. no. 33-106 (2008) and Council of Scientific and Industrial Research, India Grant F. no. 9/112 (0475) 2 K12-EMR-I. Let $z \in Z$ and $x \in N$. Then $f(z x)=f(x z)$; that is, $f(z) x+$ $z d(x)=d(x) z+x f(z)$.

\section{References}

[1] B. Hvala, "Generalized derivations in rings," Communications in Algebra, vol. 26, no. 4, pp. 1147-1166, 1998.

[2] H. E. Bell, "On prime near-rings with generalized derivation," International Journal of Mathematics and Mathematical Sciences, vol. 2008, Article ID 490316, 5 pages, 2008.

[3] H. E. Bell, "On derivations in near rings II," in Nearrings, Nearfields and K-Loops, vol. 426 of Mathematical Applications, pp. 191-197, Kluwer Academic Publishers, Dordrecht, The Netherlands, 1997.

[4] H. E. Bell and L. C. Kappe, "Rings in which derivations satisfy certain algebraic conditions," Acta Mathematica Hungarica, vol. 53, no. 3-4, pp. 339-346, 1989.
[5] H. E. Bell and G. Mason, "On derivations in near-rings," in Near-Rings and Near-Fields, vol. 137 of North-Holland Mathematical Studies, pp. 31-35, North-Holland, Amsterdam, The Netherlands, 1987.

[6] A. Asma, N. Rehman, and A. Shakir, "On Lie ideals with derivations as homomorphisms and antihomomorphisms," Acta Mathematica Hungarica, vol. 101, no. 1-2, pp. 79-82, 2003. 


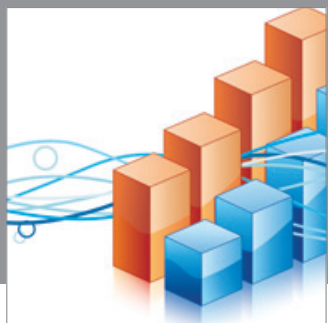

Advances in

Operations Research

mansans



The Scientific World Journal
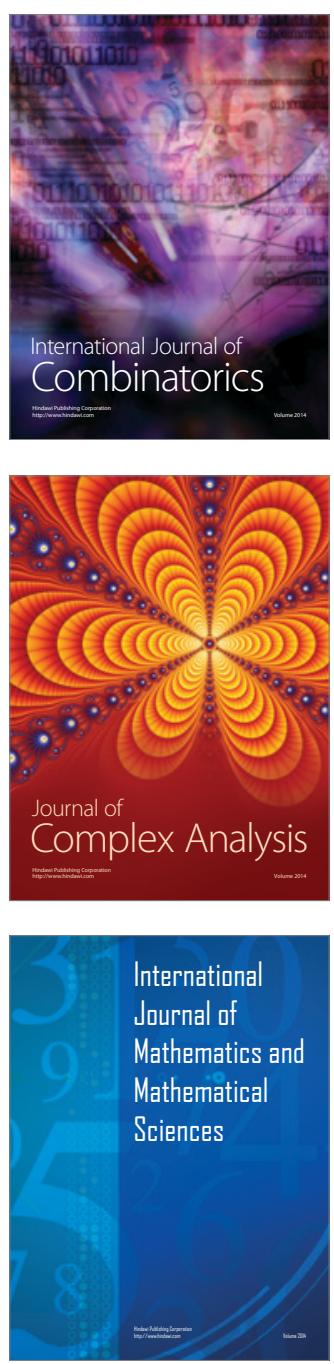
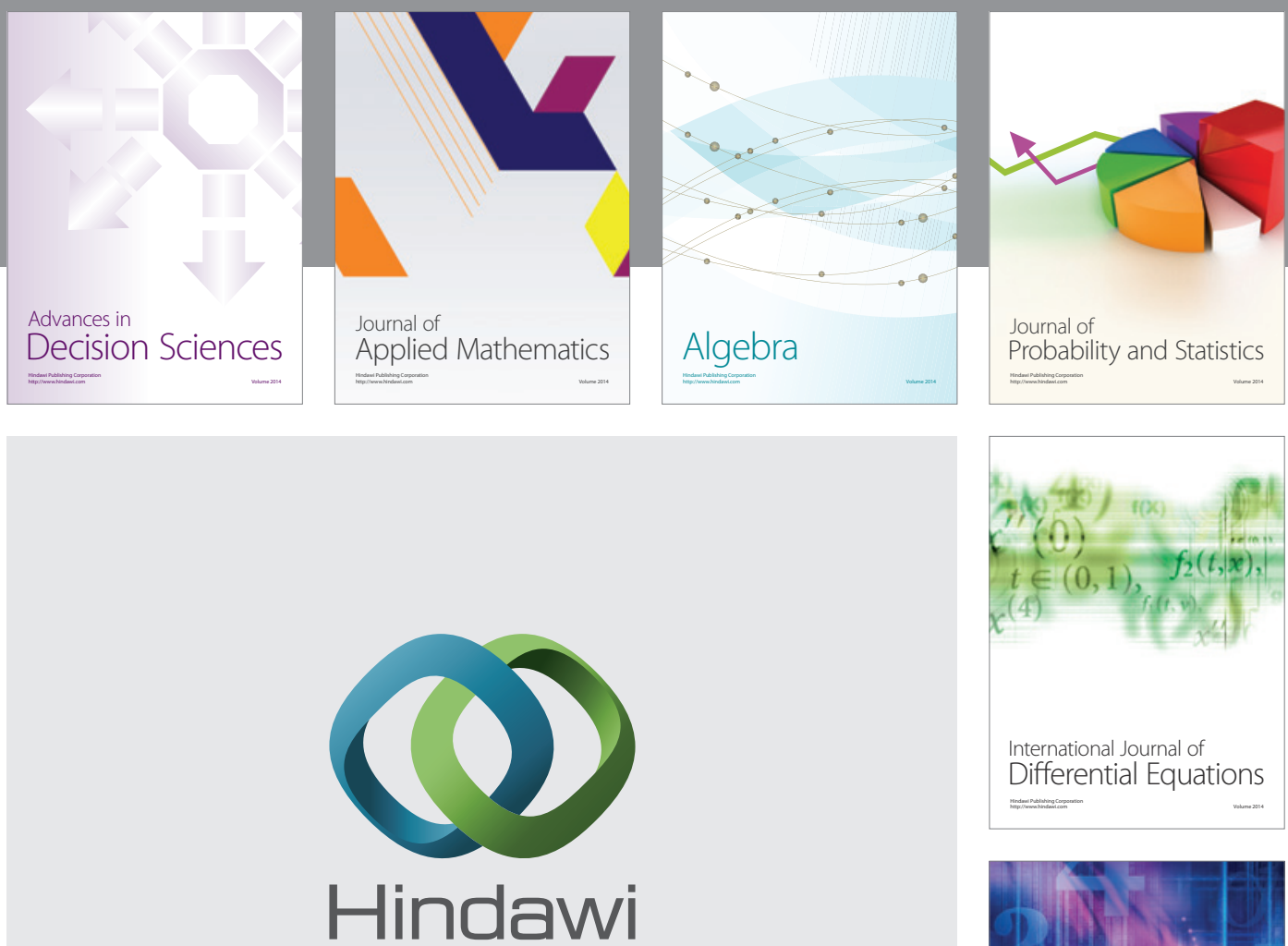

Submit your manuscripts at http://www.hindawi.com
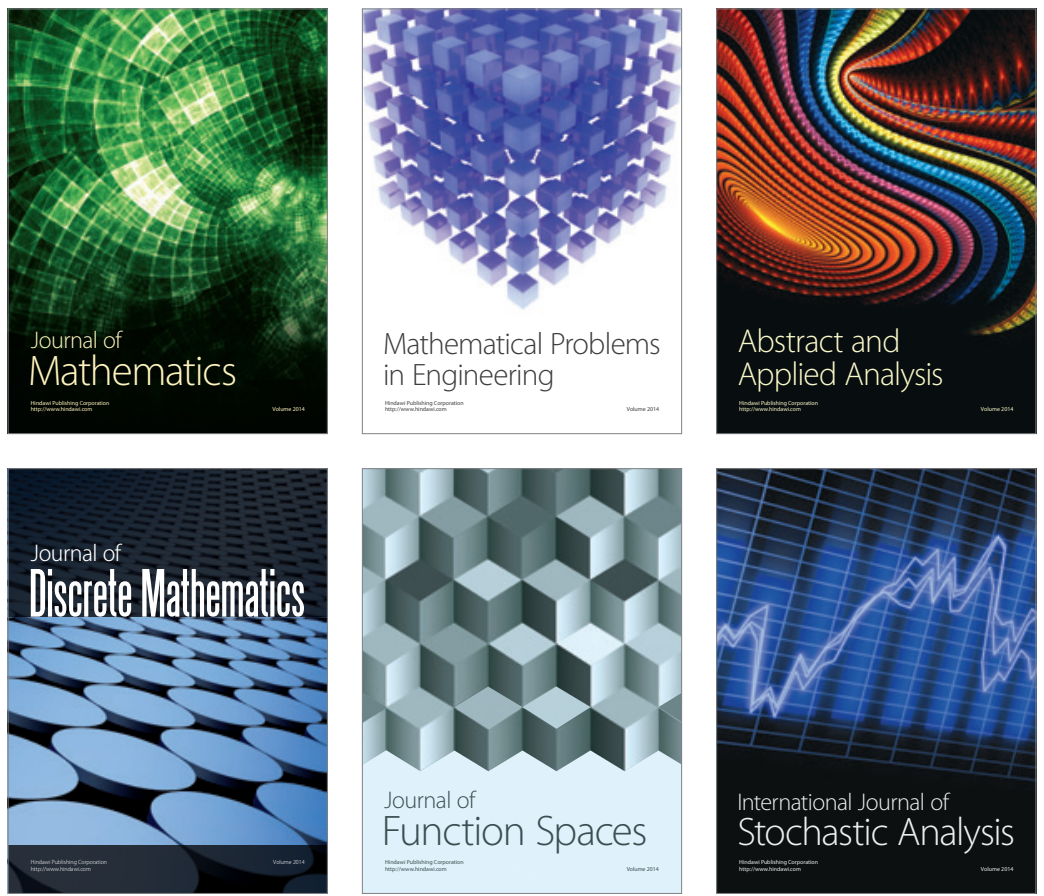

Journal of

Function Spaces

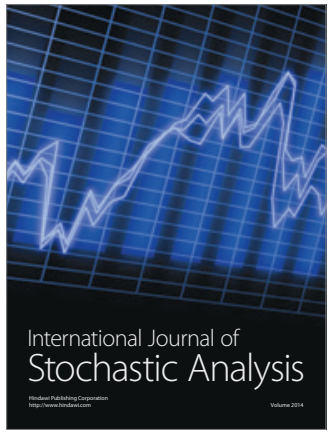

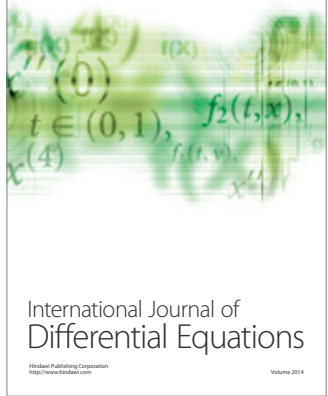
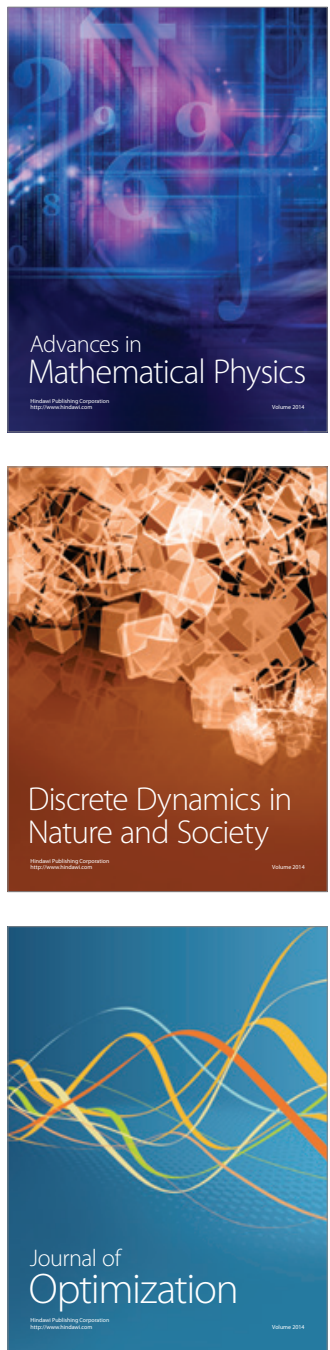